\title{
Multicolour Photometric System for Investigation of the Galaxy Population
}

\author{
V. Straižys \\ Institute of Theoretical Physics and Astronomy, Goštauto 12, Vilnius 2600, \\ Lithuania
}

\begin{abstract}
Many photometric systems for classification of stars have been proposed. However, not all of them are equally suitable for investigation of distant and reddened stars without additional information from their spectra. Earlier it was shown that the Vilnius photometric system is optimum for purely photometric classification of stars in spectral types, luminosities, metallicities and peculiarity types, when interstellar reddening is present. When realized with a CCD detector, the system permits to classify stars down to $17 \mathrm{mag}$ with a $1.5 \mathrm{~m}$ class telescope or down to $20 \mathrm{mag}$ with a $4 \mathrm{~m}$ telescope. A possibility of realizing the system with the WF camera of the Hubble Space Telescope is investigated. The problem of unification of broad-band and medium-band photometric systems is discussed.
\end{abstract}

For population study of the Galaxy in its different subsystems, very faint and reddened stars have to be classified in spectral types, luminosities and metallicities. For this one must use a sufficiently sensitive area detector and the optimum photometric system. CCD detectors are what we need. Another question: which photometric system is to be used. To be effective, such system must have bandpasses as broad as possible, the number of bandpasses must be minimum and the system must be able to classify stars in a galactic field, where objects of different temperatures, luminosity classes, populations, peculiarities and interstellar reddenings are mixed.

The optimum bandpasses of the system for two- and three-dimensional classification of stars were selected at the Vilnius Observatory in 1962-1965. This process was based on the energy distributions in the spectra of stars of different spectral types and on the interstellar extinction law. It was found that for photometric classification of stars of all spectral types, the system must include seven bandpasses at $345,374,405,466,516,544$ and $656 \mathrm{~nm}$ with half-widths of the order of $20 \mathrm{~nm}$. The eighth bandpass at $625 \mathrm{~nm}$ was also found to be useful (but not essential) for identification of M-type stars. Detailed justification of each bandpass is described in our earlier papers and in the Russian and English versions of my book "Multicolor Stellar Photometry" (Straižys, 1977, 1992). Here the functions of every bandpass will be explained briefly.

The bandpass at $345 \mathrm{~nm}$ measures the continuum intensity beyond the Balmer jump. The bandpass at $374 \mathrm{~nm}$ measures the common absorption by higher members of the Balmer series and is very sensitive to luminosity of early-type stars. The 
bandpass at $405 \mathrm{~nm}$ measures the continuum intensity before the Balmer jump for early-type stars and the metallic-line blanketing for later-type stars. The bandpass at $466 \mathrm{~nm}$ is placed near the break-point of the interstellar reddening law and is very important in distinguishing the temperature and the interstellar reddening. The bandpass at $516 \mathrm{~nm}$ measures the absorption intensity of $\mathrm{Mg}$ II triplet lines together with the $\mathrm{MgH}$ band. This bandpass is very important as a discriminator of luminosities of G5-K-M stars. The bandpass at $544 \mathrm{~nm}$ measures the continuum at the mean wavelength of the $V$ bandpass of the $B V$ system. It helps to transfer directly the magnitude scale from $B V$ to the Vilnius system. The bandpass at $656 \mathrm{~nm}$ is placed on the $\mathrm{H} \alpha$ line measuring its absorption or emission intensity for early-type stars or the pseudocontinuum for late-type stars. The bandpass at $625 \mathrm{~nm}$ measures the absorption intensity of the $\mathrm{TiO}$ band and is useful for recognition of M-type stars. Not all bandpasses are always necessary. For two-dimensional classification of earlytype stars the bandpasses at $345,374,405,466$ and 516 (or 544) $\mathrm{nm}$ are sufficient. For two-dimensional classification of late-type stars only the bandpasses at 405, 466, 516,625 and 656 may be used. If we know which stars are of spectral class $M$, the bandpass at $625 \mathrm{~nm}$ may be omitted.

The system makes it possible not only to classify normal stars with the accuracy of \pm 1 spectral subclass and to determine $M_{V}$ with the accuracy of $\pm 0.5 \mathrm{mag}$ but also to recognize Be, Am, Ap stars, subdwarfs, metal-deficient giants, carbon-rich stars, white dwarfs, horizontal-branch stars, T Tauri and Herbig Ae/Be stars, as well as many combinations of unresolved binaries.

The Vilnius photometric system has been so far successfully applied to investigate fields of the Galaxy, open and globular clusters, star forming regions, different types of peculiar stars, etc. More than 7000 stars have been observed photoelectrically down to $V=14 \mathrm{mag}$ (Straižys et al., 1989; Straižys and Kazlauskas, 1992). The system also was realized with a CCD camera on the $90 \mathrm{~cm}$ telescope of the Kitt Peak Observatory (Boyle et al., 1990a,b, 1991; Smriglio et al., 1991). Using the exposure times of the order of $5 \mathrm{~min}$ for five filters in the visible and about $20-30 \mathrm{~min}$ in the ultraviolet we were able to classify stars down to $17 \mathrm{mag}$. Even fainter stars can be reached, when using more transparent interference filters and increasing exposure time. It seems that there is no difficulty to accomplish two- and three-dimensional classification of stars down to $18 \mathrm{mag}$ with a 1.5 meter class telescope or down to 20 mag with a 4 meter telescope, with exposure times of the order of 1 hour in the ultraviolet. Exposures in all 7 colours for one field can be obtained during one night.

Very faint stars in crowded regions of the Milky Way can be observed from outside the atmosphere. The Wide-Field Camera of the Hubble Space Telescope contains filters which have their mean wavelengths at $336,375,413,469,517,547$ and $656 \mathrm{~nm}$, i.e. very close to the mean wavelengths of the bandpasses of the Vilnius photometric system. However, four of them at $375,469,517$ and $656 \mathrm{~nm}$ are much narrower. This HST system gives a possibility to classify stars in spectral and luminosity classes, as it is shown by Straižys and Valiauga (1992) using reddening-free $Q, Q$ diagrams with different combinations of bandpasses. Of course, we do not recommend to use the 
HST with the present Wide-Field Camera for stellar classification but we show that this is possible in principle and may be useful in the future.

Speaking about the HST, we must remember that in the first WFC all existing photometric systems have been neglected. This prevents using the ground-based standards of the systems and their calibration. Surely, there is no necessity to place into the HST the filters of all ground-based photometric systems. However, it would be important having there the filters at least of two systems: a "UBVRI-like" system and a medium-band system suitable for photometric classification of stars. For this I suggest to form a working group with the representatives of all most widely used photometric systems and to decide which filters are necessary for optimum photometric classification both from the ground and from the space. Introduction of such a system would help to make economy of the observing time and to increase our knowledge about the Galaxy.

The first step in this direction has been done by the VilGen system (Straižys et al., 1982, North et, al., 1982) which combines the best properties of the Vilnius and Geneva systems. A revision of the $U B V$ system proposed by Straižys $(1973,1983)$ was also realized, and 13600 stars down to $V=7.2$ have been measured in the WBVR system (Khaliullin et al., 1985; Kornilov, 1992). The magnitude $W$, replacing the ill-defined Johnson's $U$ magnitude, has an exactly known response function without red leak, and the $W-B$ indices are correctly transformed outside the atmosphere with the extinction coefficient dependent on energy distribution functions of stars (Moshkalev and Khaliullin, 1985). Realization of the $W B V R$ system on the HST would be one of the first steps towards the unification of photometric systems. Such systems (both broad- and medium-band ones) must be defined by exact response functions, not by a list of standard stars as it was the case with the $U B V, R I$ and $J H K L M N$ systems.

\section{References:}

Boyle, R.P., Smriglio, F., Nandy, K. and Straižys, V. 1990a, A\&AS, 84, 1.

Boyle, R.P., Smriglio, F., Nandy, K. and Straižys, V. 1990b, A\&AS, 86, 395.

Boyle, R.P., Dasgupta, A.K., Smriglio, F., Straižys, V. and Nandy, K. 1991, A\&AS, in press.

Khaliullin, Kh., Mironov, A.V. and Moshkalev, V.G. 1985, Ap\&SS, 111, 291.

Kornilov, V.G. 1992, in Stellar Photometry, IAU Coll. No. 136, Book of Abstracts, Dublin.

Moshkalev, V.G. and Khaliullin, Kh.F. 1985, AZh, 62, 393 = Soviet Astron., 29, 227.

North, P., Hauck, B. and Straižys, V. 1982. A\&A, 108, 373.

Smriglio, F., Nandy, K., Boyle, R.P., Dasgupta, A.K., Straižys, V. and Janulis, R. 1991, A\&AS, 88, 87 .

Straižys, V. 1973, A\&A, 28, 349.

Straižys, V. 1977. Multicolor Stellar Photometry, Mokslas Publishers, Vilnius, Lithuania. 
Straižys, V. 1983, Bull. Inform. CDS, Strasbourg, No. 25, 41.

Straižys, V. 1992. Multicolor Stellar Photometry, Pachart Publ. House, Tucson, Arizona.

Straižys, V., Jodinskienè, E. and Hauck, B. 1982. Bull. Vilnius Obs., No. 60, 50.

Straižys, V., Kazlauskas, A., Jodinskienè, E. and Bartkevičius, A. 1989. Results of Photoelectric Photometry of Stars in the Vilnius Photometric System (magnetic tape). Bull. Inform. CDS, Strasbourg, No. 37, 179.

Straižys, V. and Kazlauskas, A. 1992. Results of Photoelectric Photometry of Stars in the Vilnius Photometric System (magnetic tape).

Straižys, V. and Valiauga, G. 1992, Baltic Astron., 1, No. 4 (in press).

\section{Discussion}

A.J. Penny: $A$ limitation on using the HST for n-dimensional classification may be the low accuracy.

Straizys: Of course the accuracy of photometry obtained with the WF camera of the HST must be of the same order as for the ground-based photometry if we want to classify very faint stars in two or three dimensions. Let us hope that the present problems with low accuracy of the WF camera will be overcome. 